\title{
High-energy pulse profile of the transient X-ray pulsar SAX J2103.5+4545
}

\author{
M. Falanga ${ }^{1}$, T. Di Salvo ${ }^{2}$, L. Burderi ${ }^{3}$, J. M. Bonnet-Bidaud ${ }^{1}$, P. Goldoni $^{1}$, A. Goldwurm ${ }^{1}$, \\ G. Lavagetto ${ }^{2}$, R. Iaria ${ }^{2}$, and N. R. Robba ${ }^{2}$ \\ ${ }^{1}$ CEA Saclay, DSM/DAPNIA/Service d'Astrophysique (CNRS FRE 2591), 91191 Gif-sur-Yvette, France \\ e-mail: mfalanga@cea.fr \\ 2 Dipartimento di Scienze Fisiche ed Astronomiche, Università di Palermo, via Archirafi 36, 90123 Palermo, Italy \\ 3 Osservatorio Astronomico di Roma, via Frascati 33, 00040 Monteporzio Catone, Italy
}

Received 29 September 2004 / Accepted 26 February 2005

\begin{abstract}
Two recent INTEGRAL papers report a timing and spectral analysis of the transient Be/X-ray pulsar SAX J2103.5+4545 at high energies (5-200 keV). In this work we present for the first time a study of the pulse profile at energies above $20 \mathrm{keV}$ using INTEGRAL data. The spin-pulse profile shows a prominent (with a duty cycle of 14\%) and broad (with a $F W H M$ of $\sim 51 \mathrm{~s}$ ) peak and a secondary peak which becomes more evident above $20 \mathrm{keV}$. The pulsed fraction increases with energy from $\sim 45 \%$ at 5-40 keV to $\sim 80 \%$ at 40-80 keV. The morphology of the pulse profile also changes as a function of energy, consistent with variations in the spectral components that are visible in the pulse phase resolved spectra. A study of the double peaked profile shows that the difference in the two peaks can be modeled by a different scattering fraction between the radiation from the two magnetic poles.
\end{abstract}

Key words. binaries: close - pulsars: individual: SAX J2103.5+4545 - X-rays: binaries

\section{Introduction}

After the discovery by Hulleman et al. (1998) and suggestion that it is a Be X-ray binary, SAX J2103.5+4545 was conclusively confirmed as such by Reig et al. (2004) who estimate a distance of $\sim 6.5 \mathrm{kpc}$. The system consists of an accreting neutron star (NS) and a BOV main-sequence donor star which shows Balmer emission lines (Reig et al. 2004). The relatively short orbital period, 12.7 days (Baykal et al. 2000), and the spin period of $\sim 358 \mathrm{~s}$ (Hulleman et al. 1998) make SAX J2103.5+4545 a peculiar Be/X-ray transient system. The source does not follow the $P_{\text {pulse }}$ versus $P_{\text {orb }}$ relation followed by all other Be X-ray binaries as initially established by Corbet (1986). The source has been extensively studied in outburst by Hulleman et al. (1998) with BeppoSAX, Baykal et al. (2000, 2002) with RXTE, İnam et al. (2004) with RXTE and XMM-Newton, Reig et al. (2004) at optical and infrared wavelengths, and by Lutovinov et al. (2003), Sidoli et al. (2004) and Blay et al. (2004) with INTEGRAL.

We present here for the first time spin phase resolved measurements at energies above $20 \mathrm{keV}$, based on hard X-ray INTEGRAL observations of SAX J2103.5+4545 during a bright outburst.

\section{Results}

The data were obtained during the AO1 INTEGRAL observation of the Cygnus region, performed from May 2 to May 32003 (52761.32-52762.43 MJD). We use data from the coded mask imager IBIS/ISGRI (Ubertini et al. 2003; Lebrun et al. 2003) with an exposure of $75.06 \mathrm{ks}$, and from the JEM-X monitor (Lund et al. 2003) with an exposure time of $33.34 \mathrm{ks}$. The data reduction was performed using the standard software and response files provided in OSA 3.0 (Goldwurm et al. 2003). We extracted 32 channel spectra for IBIS/ISGRI and 256 channel spectra for JEM-X. The spectra were analyzed with the XSPEC V. 11.3 software package (Arnaud 1996). The background subtracted ISGRI light curve for the timing analysis was reduced using dedicated software (ii_light_extract, version to be included in OSA 5.0). The ISGRI light curve per energy band was obtained through a simultaneous fit, for every $20 \mathrm{~s}$ long time bin, of all sources detected in the overall image and the background using the standard least squares method in the detector domain. For JEM-X the background-subtracted light curve was obtained using standard software (see JEM-X Analysis User Manual). 


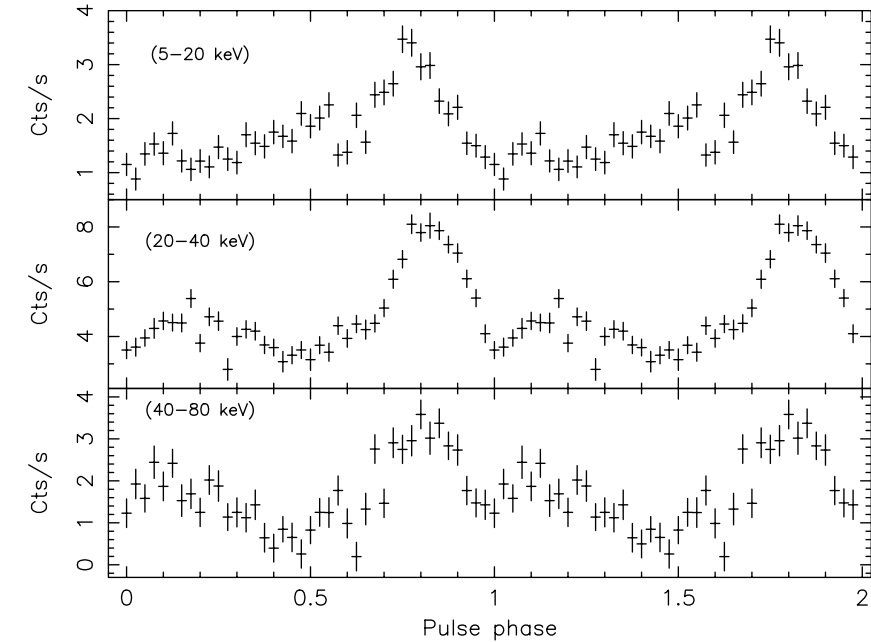

Fig. 1. JEM-X and IBIS/ISGRI light curves of SAX J2103.5+4545 folded at the NS spin period. From top to bottom, in the energy range 5-20 keV (JEM-X), 20-40 keV and 40-80 keV (IBIS/ISGRI), respectively. Each pulse profile is repeated once for clarity.

\subsection{X-ray timing}

We searched for coherent pulsations of the source in the 20-40 keV energy band where we have the best statistics. Using the $20 \mathrm{~s}$ binned light curve of the source in the 20$40 \mathrm{keV}$ energy band, we computed a Power Density Spectrum (PDS) in the frequency range between $5 \times 10^{-5}$ and $0.025 \mathrm{~Hz}$ from Fast Fourier Transforms. In the resulting PDS an evident signal is present at $v=2.8296 \times 10^{-3} \mathrm{~Hz}$, and first and second harmonics are also visible. The highest peak in the power spectrum, the first harmonic, corresponds to a nominal period of $353.41 \mathrm{~s}$. The accurate period was found using the phase-delay fitting method (see e.g., Nagase 1989) resulting in $P=353.45 \pm 0.16 \mathrm{~s}$, with an upper limit to the pulse period derivative of $|\dot{P}|<2.2 \mathrm{~s} \mathrm{yr}^{-1}$. In this section the errors are given at a $1 \sigma$ confidence level for a single parameter. The measured pulse period is in agreement with the expected value using the pulse period of $354.794 \mathrm{~s}(52761.87 \mathrm{MJD})$ and the spin-up rate of $\sim 7.4 \times 10^{-13} \mathrm{~Hz} \mathrm{~s}^{-1}$ reported by İnam et al. (2004).

Figure 1 shows the light curve folded at the best period. These profiles show a very prominent peak ( $\sim 51 \mathrm{~s} F W H M)$ around phase $\approx 0.8$. The pulse fraction (PF), defined here as $\mathrm{PF}=\left(I_{\max }-I_{\min }\right) /\left(I_{\max }+I_{\min }\right)$, increases with energy, going from $47.5 \pm 0.3 \%$ in the $5-20 \mathrm{keV}$ energy band to $45.4 \pm 6.3 \%$ at $20-40 \mathrm{keV}$ and to $80.2 \pm 8.9 \%$ at $40-80 \mathrm{keV}$. In the $80-150 \mathrm{keV}$ energy band the low signal-to-noise ratio does not allow a pulse fraction to be determined. The X-ray pulse profile of SAX J2103.5+4545 also shows an energy-dependent secondary peak around phase $\approx 0.2$, which becomes more evident at energies above $20 \mathrm{keV}$. Finally, note that the discontinuities in the top panel at phase 0.6 seem to be consistent with those observed in the pulse profile of this source by RXTE/XMM-Newton (e.g., İnam et al. 2004). Note that these discontinuities are also present in the $40-80 \mathrm{keV}$ pulse profile (bottom panel), while are not significantly present in the $20-40 \mathrm{keV}$ pulse profile (middle panel).

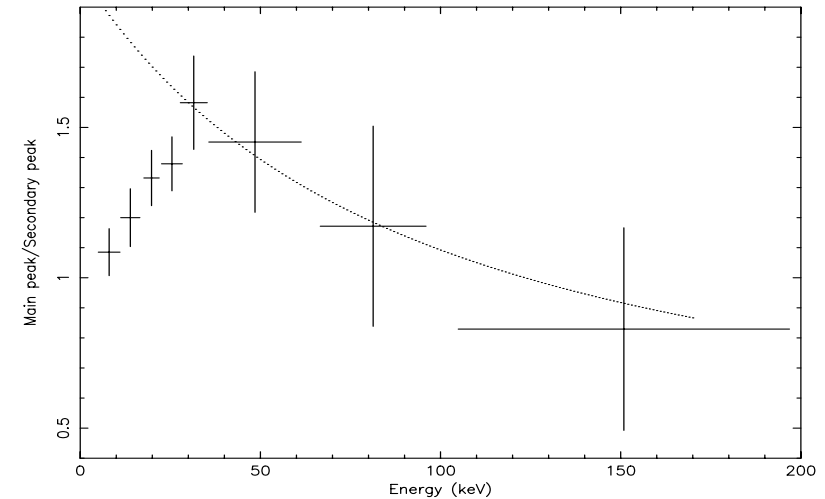

Fig. 2. Ratio of the "main" to "secondary" peak of the pulse profile as a function of energy from 5 to $200 \mathrm{keV}$. The dotted line is from the best fit two polar cap diffusion model (see Sect. 3).

\subsection{Spectral analysis and pulse phase resolved spectra}

We extracted the spectrum of the source averaged over the whole observation. In order to investigate the pulse phase dependence of the high energy emission from this source, we selected two phase intervals in the pulse profile of Fig. 1, one corresponding to the main peak $(0.5-1.0)$ and the other corresponding to the secondary peak $(0-0.5)$. From each of these phase intervals we extracted JEM-X and IBIS/ISGRI spectra. The spectral analysis has been carried out in the $5-20 \mathrm{keV}$ energy range for JEM-X and in the $20-200 \mathrm{keV}$ energy range for IBIS/ISGRI; the JEM-X spectra were rebinned to 28 channels to limit the oversampling of the instrument resolution to a factor of 3 .

The two phase-resolved spectra look different, as is evident from their ratio plotted as a function of energy in Fig. 2. The ratio gradually increases from 5 to $30 \mathrm{keV}$ and then decreases at higher energies. Note that the Direct Continuum (DC) level is not subtracted from these spectra; this might introduce a variation of the spectral ratio simply due to the fact that the DC level changes with energy. In principle we could estimate the DC level from the spectrum at the minimum of the folded light curve, although we do not know if all the emission at the minimum of the light curve is due to a DC level, or instead is due to unpulsed emission from the polar caps (this is possible for instance if parts of the polar caps are always visible). However, the statistics of these data is not good enough to extract a meaningful spectrum at the minimum of the folded light curve. Note that we took into account the presence of a DC level in the diffusion model that we used to fit this spectral ratio (see Sect. 3).

To fit the total spectrum, we first used an absorbed cut-off power law model $\left(\chi_{\text {red }}^{2}=1.28,48\right.$ d.o.f), where the photon index was found to be $\Gamma \sim 1.45$ with a cut-off at $E_{\text {cut }} \sim 30 \mathrm{keV}$. A better fit was found using an absorbed power law multiplied by a high-energy exponential cutoff model $\left(\chi_{\text {red }}^{2}=1.37,47\right.$ d.o.f. $)$, where the parameters found were similar to the ones reported in Blay et al. (2004). We then substituted this phenomenological model with a physically motivated thermal Comptonization model COMPTT, using the slab geometry (Titarchuk 1994), 


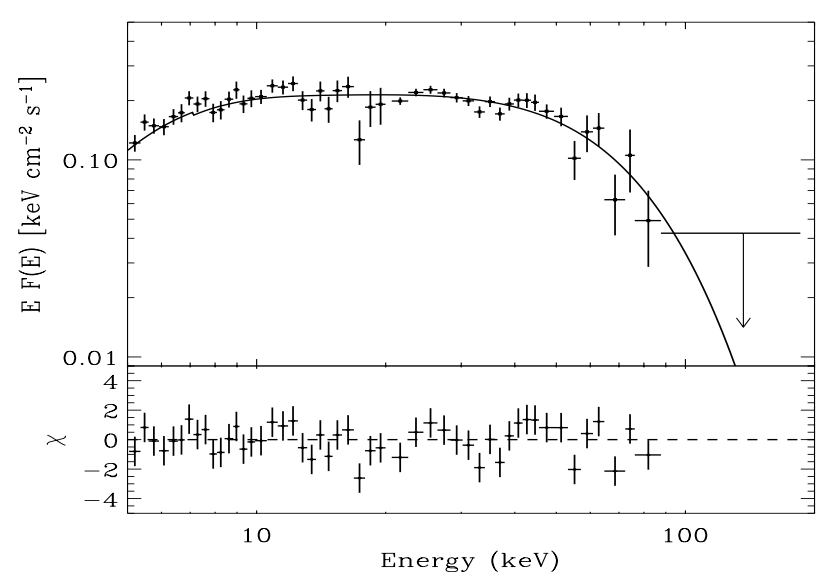

Fig. 3. The 5-200 keV unfolded spectrum of SAX J2103.5+4545 along with the best fit COMPTT model.

which gives a $\chi_{\text {red }}^{2}$ of 1.09 (47 d.o.f.). Usually the power law plus high-energy cutoff model is a good approximation of the spectra of high-magnetic field X-ray pulsars, due to the fact that the strong magnetic field modifies the Compton cross section giving a sharp cutoff in the spectrum. However, in our case, the COMPTT model provides a slightly better description of the data with respect to the "empirical" power law multiplied by a high-energy exponential cutoff on the entire dataset at a $81 \%$ confidence level (estimated by means of an F-test). We therefore prefer to report here the best-fit parameters of the COMPTT model. We interpret the parameters of the COMPTT model in the following way: at the bottom of the accretion column, i.e. at the hot spot on the NS surface, a blackbody emission, with a temperature $k T_{0}$, contributes the soft component of the spectrum, and also provides the seed photons for the Comptonization (İnam et al. 2004). These are Comptonized in the hot plasma present in the accretion column at a temperature $k T_{\mathrm{e}}$ and optical depth $\tau$.

In fitting the pulse phase resolved spectra, we found that the spectral parameters of the COMPTT model for the two spectra are compatible at a $90 \%$ confidence level. In order to better constrain the optical depth, $\tau$, for the simple diffusion model (see Discussion), we fixed the seed photons temperature $k T_{0}$ and the hot plasma temperature $k T_{\mathrm{e}}$ to a single value (see Table 1). In Fig. 3 we show the unfolded $E F(E)$ spectrum of the entire observation, plotted together with the residuals in units of $\sigma$ with respect to the best fit COMPTT model. The best fit parameters together with the $90 \%$ c.l. errors for a single parameter are reported in Table 1.

\section{Discussion}

We analyzed for the first time the shape of the pulse profile of SAX J2103.5+4545 at energies above $20 \mathrm{keV}$. The observed $\mathrm{X}$-ray pulse profile shows a broad main peak and a secondary peak that seems to become more prominent at higher energies. The differences between the spectra of the main (pulse phase: $0.5-1$ ) and secondary (pulse phase: $0-0.5$ ) peak are evident in their ratio, which increases with increasing energy up to $\sim 30 \mathrm{keV}$ and then decreases, indicating that the main peak is characterized by an excess of emission in the energy range $20-50 \mathrm{keV}$; the difference in the mean 5-200 keV luminosity between the two pulse phase resolved spectra is a factor of about 1.5. The pulsed fraction is observed to significantly and steeply increase with energy, from $\sim 45 \%$ up to $40 \mathrm{keV}$ to $\sim 80 \%$ in the range $40-80 \mathrm{keV}$. A similar behaviour was also found for the Be/X-ray transient RX J0117.6-733 where the pulsed percentage increased to at least $79 \%$ in the $20-70 \mathrm{keV}$ band (Macomb et al. 1999).

The average spectrum, as well as the pulse phase resolved spectra (i.e. main peak spectrum and secondary peak spectrum) can be well described by a Comptonization model. According to this model, the seed photons for the Comptonization have a temperature of $k T_{0} \sim 1.5 \mathrm{keV}$, injected from the bottom of the accretion shock of electron temperature $k T_{\mathrm{e}} \sim 13 \mathrm{keV}$ and Thomson optical depth $\tau \sim 2.4$. The electron temperature is too high for an accretion disk (Frank et al. 2000).

In the hypothesis that the two peaks in the pulse profile come from the two polar caps on the NS surface, the variation of the pulse profile with energy cannot be simply explained by geometrical effects (the viewing angles of the polar caps, when directed towards the observer, are different and the projected emission areas in the line of sight are different) because the geometrical effects can change the observed flux but not the spectral shape. We therefore propose that the differences between the spectra at the two peaks is mainly caused by scattering effects. Since the main peak does not vary significantly with the energy we consider in our model that only the second peak is scattered.

Considering that the radiation in the main peak comes from one polar cap and from the continuum of the NS, the flux can be written $I_{\text {spot }}+I_{\text {NS }}$. The secondary peak then is $I_{\text {spot }}^{\prime} \mathrm{e}^{-\tau^{\prime}}+I_{\mathrm{NS}}$ (part of the secondary pole emission $\left(1-\mathrm{e}^{-\tau^{\prime}}\right)$ is scattered away from the line of sight and does not contribute to the the observed peak flux). $\tau^{\prime}$ is given from the Comptonization fit parameter and from the Klein-Nishina equation, noting that in our case $k T_{\mathrm{e}} \ll m c^{2}$. Above $30 \mathrm{keV}$, the pulsed fraction is $80 \%$ which shows that the continuum from the NS has a small contribution. In that energy range, the ratio given in Fig. 2 represents the ratio of the two flux peaks, which indeed is well fitted by the simple diffusive model, $(1+\alpha) /\left(1+\alpha^{\prime} \mathrm{e}^{-\tau^{\prime}}\right)$, where $\alpha=I_{\text {spot }} / I_{\mathrm{NS}}$ and $\alpha^{\prime}=I_{\text {spot }}^{\prime} / I_{\mathrm{NS}}$ (note that $I_{\text {spot }}$ and $I_{\text {spot }}^{\prime}$ may be different due to geometrical effects, since the viewing angles of the magnetic poles, when directed towards the observer, may be different resulting in a difference in the projected emission areas along the line of sight). Although the statistics are not good enough to constrain all the parameters (the error bars on the single parameters are quite large), interestingly we always obtain that $\alpha^{\prime}=0.1 \alpha$. We can therefore quantify the geometrical difference between the two projected emission areas as $10 \%$. On the other hand, in the energy range between $5-30 \mathrm{keV}$ with a pulsed fraction of $\sim 50 \%$, the continuum of the NS contributes half of the emission. The emission of the secondary peak is likely scattered through the Compton shock layer, above the hot spot, and contributes to the continuum. 
Table 1. Best fit parameters of the absorbed COMPTT model for the spectra of SAX J2103.5+4545 together with $90 \%$ confidence errors for a single parameter.

\begin{tabular}{lcccc}
\hline \hline Pulse phase & $0.0-0.5$ & $0.5-1.0$ & All & All \\
\hline$N_{\mathrm{H}}\left(10^{22} \mathrm{~cm}^{-2}\right.$, fixed) & 3.45 & 3.45 & 3.45 & 3.45 \\
$k T_{0}(\mathrm{keV})$ & $1.5(\mathrm{f})$ & $1.5(\mathrm{f})$ & $1.50 \pm 0.17$ & $1.5(\mathrm{f})$ \\
$k T_{\mathrm{e}}(\mathrm{keV})$ & $13.0(\mathrm{f})$ & $13.0(\mathrm{f})$ & $13.68 \pm 1.85$ & 13.0 (f) \\
$\tau$ & $2.08 \pm 0.17$ & $2.5 \pm 0.13$ & $2.37 \pm 0.43$ & $2.53 \pm 0.14$ \\
$\chi_{\text {red }}^{2}$ & $1.2(49$ d.o.f.) & $1.2(49$ d.o.f.) & $1.09(47$ d.o.f.) & 1.06 (49 d.o.f.) \\
$L_{5-200 \mathrm{keV}}^{*}\left(10^{36} \mathrm{erg} \mathrm{s}^{-1}\right)$ & 2.95 & 4.64 & 4.17 & 4.21 \\
\hline
\end{tabular}

${ }^{*}$ Assuming a distance of $6.5 \mathrm{kpc}$.

Finally, from the observed double-peaked folded light curve, we infer a limit on the angles that determine the geometry of the accretion powered pulsar (if the NS has a pure centered dipole field); these are the angle between the rotation axis and the direction to the observer, $i$, and the angle between the magnetic and rotation axes, $\beta$. Assuming a typical mass value of $20 M_{\odot}$ for the BOV companion star (Reig et al. 2004) and a $1.4 M_{\odot}$ NS, from the mass function the inclination angle of the system is $i \sim 30^{\circ}$. The parameter space when two polar caps are seen is determined by the condition $i>90^{\circ}-\beta-\delta g$, where $\delta g$ is the gravitational light deflection of the emitted X-ray (Beloborodov 2002). In our case, considering $\delta g=0$, this leads to $\beta<60^{\circ}$, or higher with non-zero $\delta g$. The gravitational light bending effect is less than $10^{\circ}$ (see Bulik et al. 2003).

Acknowledgements. M.F. is grateful to P. Laurent and G. Israel for valuable discussions and to A. Gros and S. Chazalmartin for providing the light curve software. This work is supported through the CNES and the CNRS programme.

\section{References}

Arnaud K. A. 1996, in Astronomical Data Analysis Software and Systems V, ed. G. H. Jacoby, \& J. Barnesin, ASP Conf. Ser., San Francisco, 101, 17
Baykal, A., Stark, M. J., \& Swank, J. H. 2000, ApJ, 544, L129

Baykal, A., Stark, M. J., \& Swank, J. H. 2002, ApJ, 569, 903

Blay, P., Reig, P., Martínez, et al. 2004 [arXiv: astro-ph/0407580]

Beloborodov, A. M. 2002, ApJ, 566, L85

Bulik, T., Gondek-Rosińska, D., Santangelo, A., et al. 2003, A\&A, 404, 1023

Corbet, R. D. 1986, MNRAS, 220, 1047

Frank, J., King, A. B., \& Raine, D. 2002, Accretion Power in Astrophysics (Cambridge University Press), 3rd ed.

Goldwurm, A., David, P., Foschini, L., et al. 2003, A\&A, 411, $\mathrm{L} 223$

Hulleman, F., in 't Zand, J. J. M., \& Heise, J. 1998, A\&A, 337, L25

Lebrun, F., Leray, J., Lavocate, P., et al. 2003, A\&A, 411, L141

İnam, S. C., Baykal, A., Swank, J. H., \& Stark, M. J. 2004, ApJ, submitted [arXiv: astro-ph/040221]

Lund, N., Budtz-J $\phi$ rgensen, C., Westergaard, N. J., et al. 2003, A\&A, 411, L231

Lutovinov, A. A., Molkov, S. V., \& Revnivtsev, M. G. 2003, AstL, 29, $\mathrm{L} 713$

Macomb, D., Finger, M. H., Harmon, B. A., et al. 1999, ApJ, 518, L99

Nagase, F. 1989, PASJ, 41

Reig, P., Negueruela, I., Fabregat, J., et al. 2004, A\&A, 421, 421

Sidoli, L., Mereghetti, S., Larson, S., et al. 2004, in the 5th INTEGRAL Workshop [arXiv: astro-ph/0404018]

Titarchuk, L. 1994, ApJ, 434, 570

Ubertini, P., Lebrun, F., Di Cocco, G., et al. 2003, A\&A, 411, L131 